\title{
Demonstration of a 10-m Solar Sail System
}

\author{
David M. Murphy ${ }^{*}$ and Brian D. Macy ${ }^{\dagger}$ \\ ABLE Engineering, Goleta, CA 93117 \\ James L. Gaspar \\ NASA Langley Research Center, Hampton, VA 23681
}

\begin{abstract}
The NASA In-Space Propulsion (ISP) program has been sponsoring system design development and hardware demonstration activities of solar sail technology over the past 16 months. Efforts to validate by test a moderate-scale (10-m) 1/4-symmetry ground demonstration sail system are nearly complete. Results of testing and analytical model validation of component and assembly functional, strength, stiffness, shape, and dynamic behavior are discussed.
\end{abstract}

\section{Introduction}

$\mathrm{N}$ o large-scale technologies closely related to solar sailing have yet been demonstrated successfully in space. Over the past decades many small disparate activities in solar sail technology development have been pursued. As a result, materials technology, fabrication experience, and applicable analytics have been brought forward to a point where projections for system performance have begun to have real credibility.

In 2001 the ISP Program, managed by the Office of Space Science at NASA Headquarters, determined that the time was right to pursue a system demonstration of sail technology that would elevate the Technology Readiness Level of solar sailing sufficiently to allow flight implementation. NASA embarked on a competitive, gated multiyear program, implemented by the ISP Projects Office at Marshall Space Flight Center (MSFC), to pursue the development and ground demonstration of system-level sail technology.

Under a 30-month NASA ISP program, AEC-Able (ABLE) Engineering, in concert with other activities ${ }^{1}$ also under the purview of the ISP projects office at MSFC, is developing scalable analytical tools and design technologies for a solar sail propulsion system. The subject ISP Ground System Demonstrator development and validation effort, led by ABLE Engineering, is performed with the assistance of the Systems Technology Group of SRS Technologies (sail assembly provider), the Langley Research Center (LaRC) for sail shape modeling and dynamics testing, and the MSFC Space Environmental Effects Laboratory (materials characterization and life evaluation).

In the first phase of the program (6 months) activities were focused on design and analysis refinement of the still-evolving Scalable Square Solar Sail $\left(\mathrm{S}^{4}\right)$ concept $^{2}$ and refinement of plans for hardware development and demonstration ${ }^{3}$ in Phases 2 and 3. The on-going Phase 2 effort encompasses design, fabrication, and validation-through a series of component and system tests-of a $10-\mathrm{m}$ (1/4-symmetry) demonstration system, shown in Figure 1. Validation activities culminate with the demonstration of deployment, and sail shape and system dynamics measurement (in vacuum at LaRC).

\footnotetext{
${ }^{*}$ Chief Research Engineer, ABLE Engineering Co. Inc., 7200 Hollister Avenue, Goleta CA 93117, Member AIAA.

${ }^{\dagger}$ Technical Director, ABLE Engineering Co. Inc., 7200 Hollister Avenue, Goleta CA 93117.

${ }^{*}$ Research Engineer, Structural Dynamics Branch, NASA Langley Research Center, 4 West Taylor Street, MS 230, Hampton, VA 23681, AIAA Member.
} 
In this paper some of the recent design developments important to the functionality of the 10-m system hardware are reviewed first. Critical validations for key aspects of the system are emphasized. The performance of the hardware thus far in test is reviewed and compared to analytical predictions. Lessons learned in the demonstration and test of the 10-m system are being integrated into ongoing design and analysis activities in support of the build and test of a 20-m full (4 quadrant) sail system in Phase 3.

\section{Demonstration and Validation}

The ISP projects office at NASA headquarters has prioritized the maturation of solar sail propulsion technologies in order to enable or enhance a variety of space science missions. ${ }^{4}$ Solar sails, especially as a system, present complex engineering challenges. In particular, the difficulty of validating modeling results-through testing in a fully representative environment prior to flight-has thus far discouraged favor of this enabling propulsion technology by near-term mission planners.

The system demonstration and validation activities discussed herein will systematically reduce the risk of flight implementation through a series of demonstrations of increasingly more complex solar sail systems and testing of the highest fidelity possible in the terrestrial environment. In our ongoing (Phase 2) activities (and into Phase 3), analytical and computer models are being refined and correlated as testing progresses on larger and more highly engineered sail systems. First, CP1 sails and a prototype graphite Coilable were assembled and tested. Over the past 10 months a 1/4-symmetry deployable system 10 meters in size has been built and tested. In the next year a $20-\mathrm{m}$ full $\mathrm{S}^{4}$ system will be built and validated with thorough ground testing. Deployments will be performed in the 100-ft-diameter

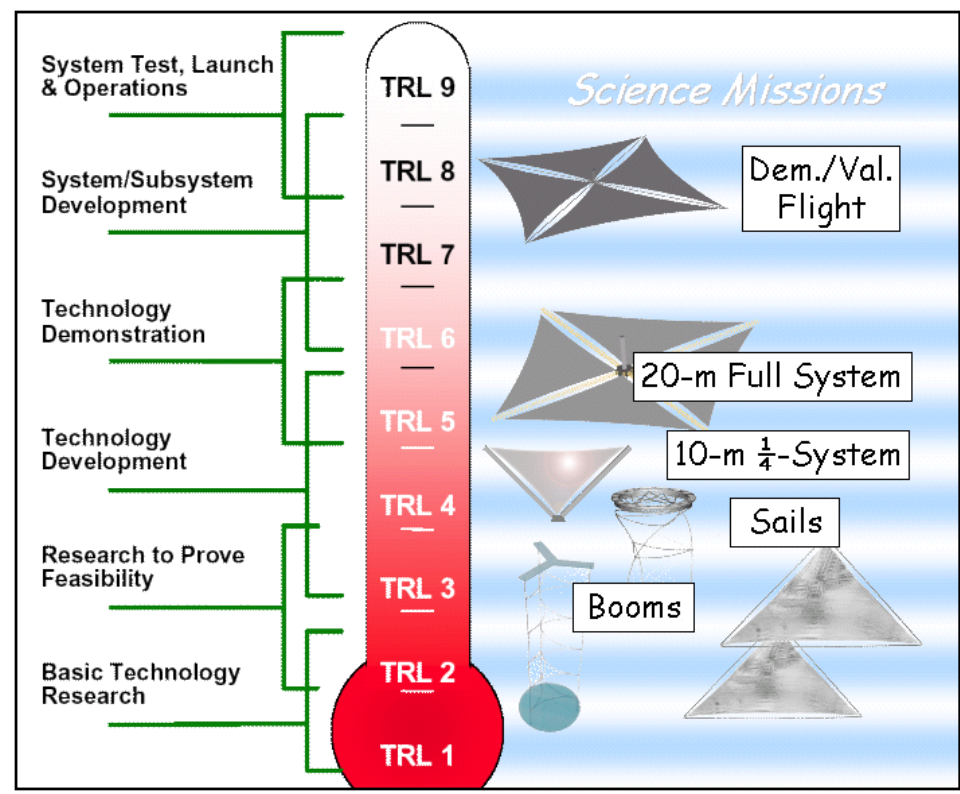

Figure 2. Advancing Technology Readiness Level thermal vacuum chamber facility at the NASA Glenn Research Center Plum Brook facility. As shown in Figure 2, these activities will elevate the Technology Readiness Level (TRL) sufficiently to where a validation flight is warranted.

The rationale for the necessity of a flight validation is based in large part on the unsuitability of the ground environment for deployment testing. The deployment kinematics of a Coilable mast are controlled, predictable, and linear. But a large ultra-thin membrane presents difficult challenges in ensuring a predictable, repeatable deployment, especially with the forces of gravity. The examination of the deployed system is also hampered in a gravity environment. The gravity-free shape of the sail, as well as the mast, is critical to performance. For the sail, the surface topology at low stress levels is affected strongly by numerous imperfection sources. ${ }^{3}$ For a gossamer mast, the local and global waviness can significantly reduce the strength and stiffness performance.

Given realities such as these, the reticence of mission planners to adopt solar sails is understood. Yet through the scope of work now in progress under the auspices of the NASA ISP program, these and other known and as-yet unknown issues in sail systems are being rigorously investigated. And when these efforts are complete a fully validated, scalable, mission-enabling propulsion system will be ready for flight demonstration.

\section{System Description}

The current $\mathrm{S}^{4}$ concept integrates gossamer Coilable mast and sail membrane technology, solar arrays, launch tie-down and release mechanisms, and attitude control actuators, efficiently packaged within structure shared by other bus components and mission payloads to form a generic scalable sailcraft possessing reliable deployment, structural robustness and determinate sail shape with-most critically—minimized overall mass and volume. Views of a (truncated structure, scaled sail) 40-m S${ }^{4}$ sailcraft system are shown in Figure 3. 


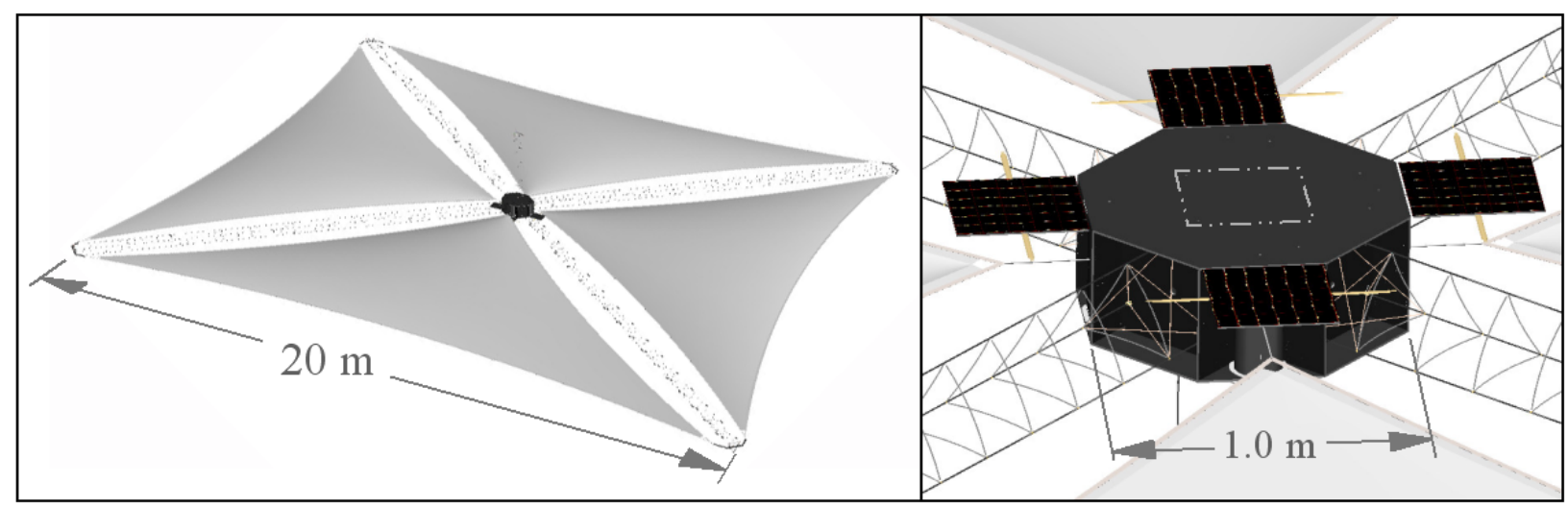

Figure 3. Depiction of a 20-m S4 System with Detail of the Central Assembly

The 10-m S $\mathrm{S}^{4}$ ground system (shown in Figure 1) consists of three major subassemblies: The Sail Assembly, the Mast Assembly (qty. 2), and the Central Assembly. The 7-m carbon composite mast (diameter of $40 \mathrm{~cm}$ ), which is a truncated length of structure optimized for an $80-\mathrm{m}$ sail system, possesses a $70 \mathrm{~g} / \mathrm{m}$ linear mass. The sail is composed of an aluminized 3-micron CP1 membrane with integral shear compliant borders and graphite edge cords.

The sail is connected to structure at three points, which provides for a deterministic structural loading condition and assures a planar sail shape. The sail and mast designs are flight worthy constructions (in design, materials, and fabrication) and are readily scalable to a 40-m or 80-m or larger system. The central assembly is a low fidelity aluminum structure, which serves as a housing for the stowed masts and sail, and the mechanism necessary for their deployment. In the next phase of the program a flight-representative central structure, and additional mechanism (e.g. ACS actuator systems ${ }^{5}$ ) will be integrated with larger sails and longer masts to complete a $(20-\mathrm{m})$ full $\mathrm{S}^{4}$ sailcraft system.

\section{A. Overview}

\section{Ambient Testing Program}

In order to reduce programmatic risks associated with system testing, engineering development unit (EDU) assemblies of the mast and sail designs were constructed and tested first. The first EDU mast was constructed of offthe-shelf composite members. This unit incorporated new gossamer concepts for the 2DOF joint and structural interface of the batten and diagonals into the longeron fitting. This "node" design, which was also optimized for mass production, proved unsatisfactory in strength and kinematic reliability. A second EDU (10 bays in length) was built with fittings derived from flight heritage masts, but sized much smaller and composed mostly of magnesium. The kinematic function and strength of these fittings proved robust, as did the new custom-pultruded IM9 graphite composite structural members (longerons and battens).

The linear mass of this mast design is $70 \mathrm{~g} / \mathrm{m}$. Compared with the lightest heritage satellite boom system, ${ }^{\S}$ the ISP mast is $25 \%$ lighter, $31 \mathrm{X}$ stiffer and $6 \mathrm{X}$ stronger due to the incorporation of high-performance graphite longerons and battens. The validation of the functionality of this new gossamer sail mast design provided the confidence to proceed with the fabrication of the two 7-m (31-bay) masts for the 10-m Quadrant Assembly.

In efforts scheduled in parallel with the EDU masts, SRS Technologies was developing GSE and processes for assembly of the sails. The first ISP-program sail incorporated 5-micron sail material, adhesiveless seaming processes and new border and cord designs. This sail assembly, named the Workhorse Sail, was critical for the timely development of packaging and deployment methods.

Various further developments in design and fabrication methods were incorporated on a second 5-micron sail. In particular, the sequencing elements developed on the Workhorse (to provide a highly managed deployment) were incorporated. This Refined Sail (RS5) and a third sail (RS3) incorporating further refinements and 3-micron film were both used for system level test and modeling correlation, as is described later.

The mast and sail assembly testing was structured generally as shown in Figure 4 below. The particulars of mast testing and sail testing are discussed next, followed by integrated system testing plans and results to date.

\footnotetext{
${ }^{\S}$ IMAGE Coilable metrics: Diameter $=18.5 \mathrm{~cm}, \mathrm{~L}=10 \mathrm{~m}$, linear mass $=93 \mathrm{~g} / \mathrm{m}, \mathrm{EI}=2,660 \mathrm{~N} \cdot \mathrm{m}^{2}, \mathrm{M}_{\mathrm{cr}}=8.5 \mathrm{~N} \cdot \mathrm{m}$
} 


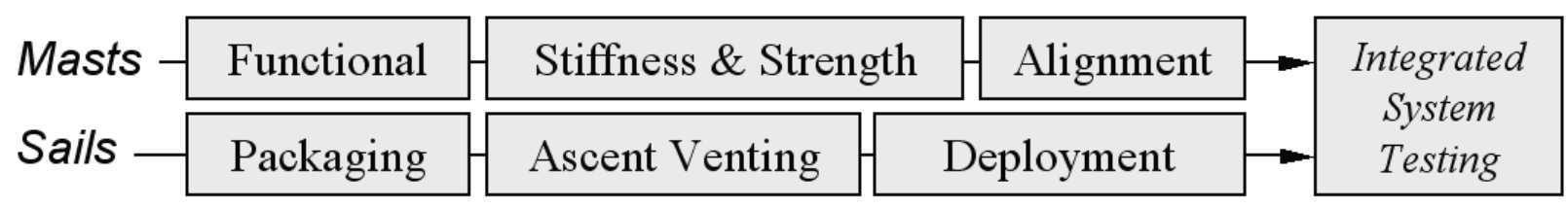

Figure 4. Mast and Sail Assembly Test Flow

\section{B. Mast Testing}

The first evaluation of the completed 7.1-m mast assemblies was a check of stowage and deployment. The masts stowed properly into a 6 -cm-high coil, meeting the expected linear compaction factor $(0.85 \%)$. The mast deployments were performed horizontally with an overhead rail car for tip off-loading. The masts self-deploy and are rate-limited by an axially-located lanyard and a DC motor. The internal strain energy of the coiled longerons and buckled battens provides a push force measured at $7 \mathrm{lbs}$, as was predicted by simple closed form calculations.

Basic functional performance had already been demonstrated on EDU2. At issue with these masts were structural strength and stiffness identification prior to further integration activities, such as harness routing and mounting within the central structure. Testing each mast alone, on a rigid base, was advantageous for precise model correlation.

Stiffness and strength were evaluated using lateral tip deflections imparted by a simple setup consisting of a pulley and various weights. The deflections were measured using a laser tracking system that followed a corner cube mounted on the mast tip ground support equipment (GSE). As this mast design is self-supporting at this length, free vibration could also be documented with the laser tracker. The first bending mode (with a tip assembly plus GSE mass of $0.7 \mathrm{lbs}$ ) can be calculated fairly precisely from averaging the vibrations over the first 10 cycles (see Figure 5).

The bending stiffness cannot be measured directly as one third of the compliance under lateral tip loading is due to mast shear stiffness (GA). A pretest FEA model of the mast, which captures shear and bending compliance, predicted a first mode of $6.1 \mathrm{~Hz}$. The tip mass during the test was $0.1 \mathrm{lbs}$ heavier, so this agreement was considered

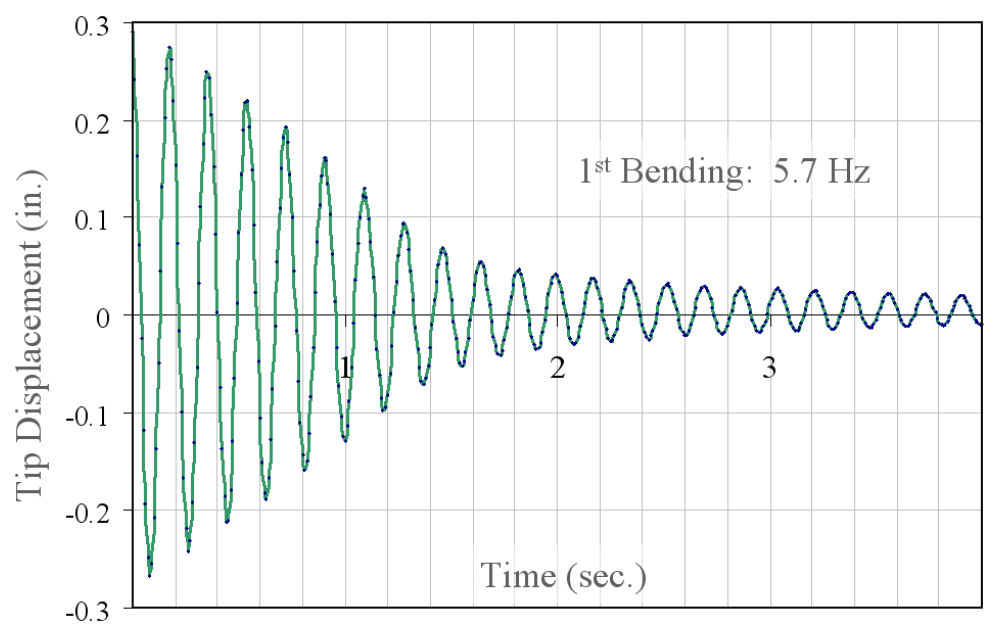

Figure 5. Mast Frequency Test Data, Cantilever Mode adequate. Further testing, to be performed by LaRC, was planned to capture more modes and well as the actual damping (which was significantly affected by air drag in this test).

The close agreement between the estimated and measured first mode demonstrates that the Coilable is generally a very predictable, readily-modeled, linear structure. However, longer masts and or substantially lighter designs are susceptible to stiffness, and hence also strength, reduction due to local and global waviness. ${ }^{6}$

Modeling which incorporates these effects, which can be coupled, is not trivial and is still incomplete. The asmanufactured waviness is critical to accurate modeling. To provide data for such models, to support large sail system design, the straightness of the assembled masts was measured. A laser tracker ball was suspended from a small hook that hung from a longeron. This target was moved from bay to bay along the longeron of a (cantilevered) mast.

Due to necessities of schedule, the masts were at this time integrated with the central structure and fitted with harnessing on their lower longeron. Consequently only the two top longerons on each mast were measurable. Data for the vertical ${ }^{\S}$ displacement along each longeron (4 total) is shown in Figure 6, where "droop" of the cantilever has been removed.

\footnotetext{
$\S^{\S}$ Close examination of the point-to-point lateral waviness data reveals friction from the hook was preventing good target position repeatability.
} 


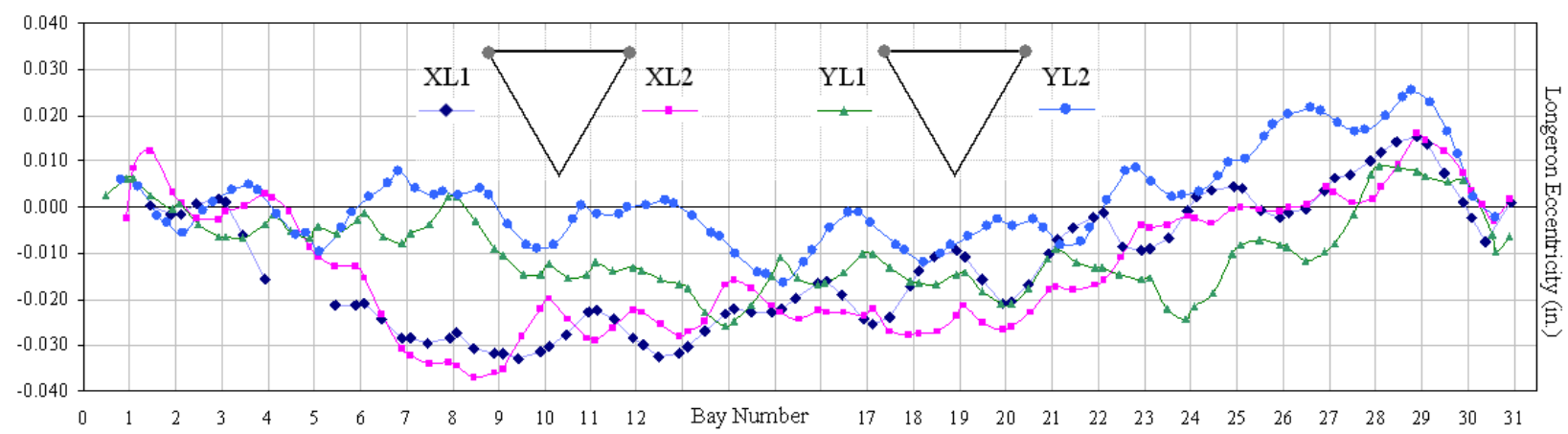

Figure 6. Longeron Straightness Data for X and Y Masts (Top Longerons)

Each bay was measured in 3 places, near the midpoint and beside the fittings on either end. The two values from either side of a given fitting were averaged and the data for variation in straightness from fitting node to bay midpoint (4.5-in. spacing) were compiled into a histogram, shown in Figure 7. The standard deviation of this (planar) data is 4 mils. Up to this point in sail mast design a worst cast variation of 7 mils had been assumed. Even the worst case observed eccentricity leads to a negligible correction to performance at the design length and load (40-cm mast and a $80-\mathrm{m}$ sail system at $1 \mathrm{psi}$ ).

Three-dimensional waviness data, measured continuously along the length, is currently being collected on a more gossamer $(34 \mathrm{~g} / \mathrm{m})$ mast under a complimentary NASA program (New Millennium ST8, The SAILMAST Experiment). The ST8 mast is shown in Figure 8, alongside a comparable diameter heritage S2 glass (section of 50-m-long LACE boom) and the ISP 10-m Quadrant mast. These data sets, and the test and analysis planned under the ST8 program, will allow predictive capability for long slender sail mast strength and stiffness

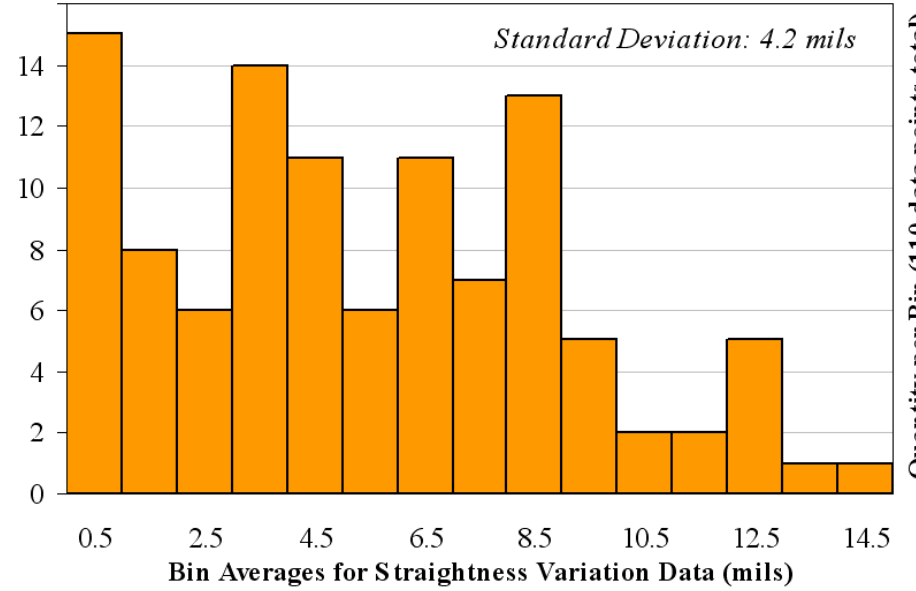

Figure 7. Histogram of Mast Straightness Data performance.

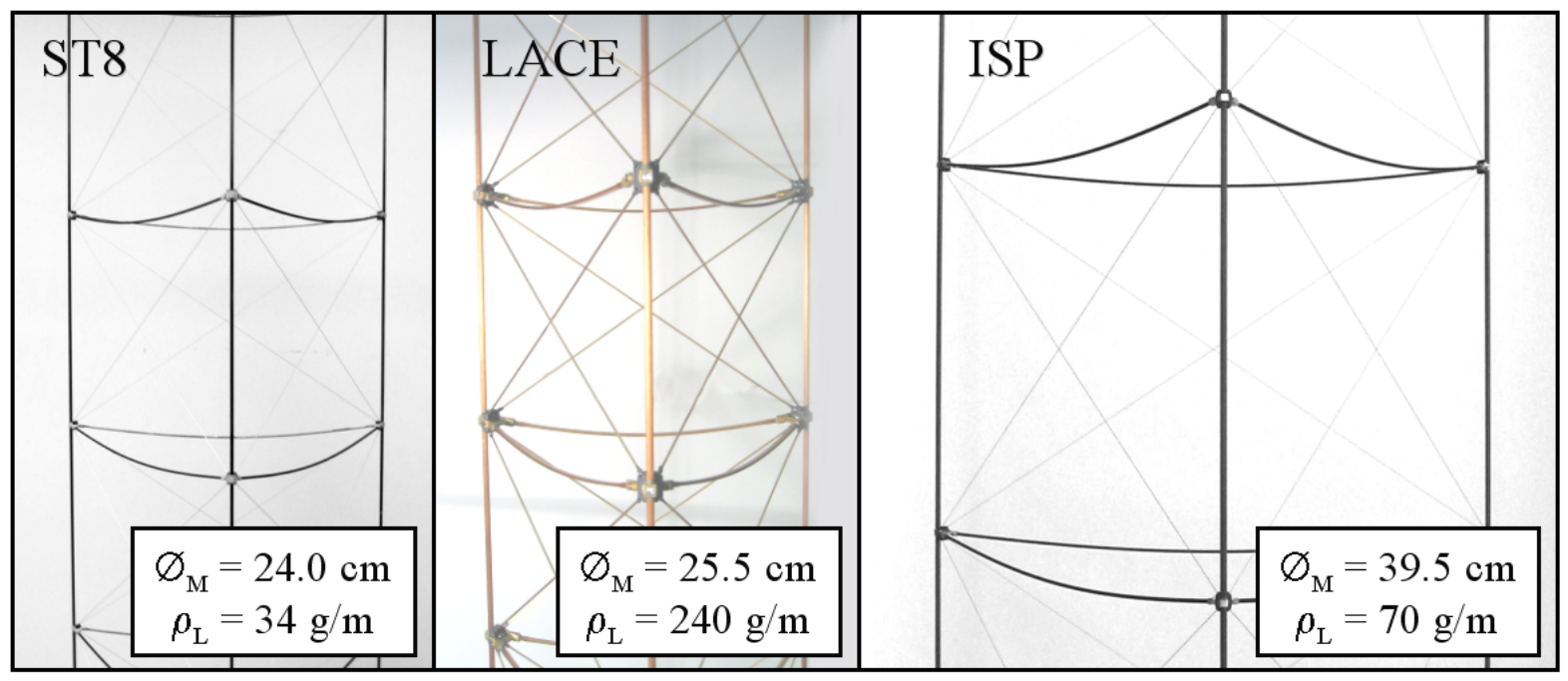

Figure 8. Comparison of a Heritage S2 Glass Boom to Gossamer Sail Masts 


\section{Sail Testing}

The first challenge for testing of the sail assembly was packaging into the storage volume and integration with the deployment management mechanism. When the Workhorse Sail was first folded it was apparent that attempts to combine low-mass launch restraint clips and an imprecisely folded sail were incompatible: The clips were too small to accommodate fold-to-fold variations.

It was necessary to develop GSE for precise, repeatable folding. The baseline folding scenario called for 15 -in. folds parallel to the long edge, followed by 5 -in. folds-in from each corner-to result in flat packs of the two halves of the sail. The primary folding was accomplished fairly readily. The sail was rolled onto a 10-m-long thinwalled aluminum cylinder. The rolling process begins with the inboard corner and can be done without precision. The next step is to bring the long edge (hypotenuse) down off the cylinder and position the cord along a matching curve marked on the folding table. The cylinder was positioned over a 9-m-long folding table, supported with rollers to allow free rotation. The sail was then folded back and forth onto the table at precise 15 -in. increments using tensioned steel tapes to enforce the fold lines. The secondary folding proved to be more problematic to perfect, but once a satisfactory method was developed, the sail could be stowed with enough precision to use the as-built stowage clips.

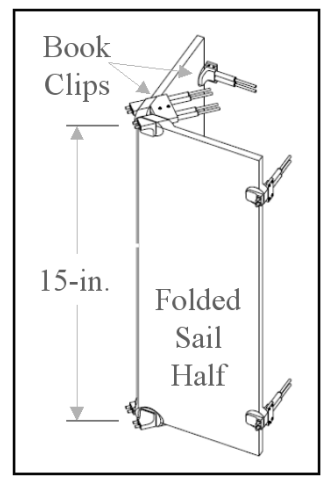

The design of the clips involved a stack of thin Teflon sheets (1-in. in diameter) interleaved with the sail folds at 4 positions around the perimeter of each sail half-stack. The stacks were positioned such that a portion of the Teflon stack overhung the sail stack edge and there they were bound together with parallel rods for mutual alignment. Rigid "bookends", one free to slide along the rods and preloaded by a spring, squeezed the book of Teflon pages (and interleaved sail edges) locally. This arrangement was intended to enforce sequential deployment of the 5-in. folds, with the book allowing only the top layer to slip out as each half of the sail is pulled out alongside the mast.

Activities for refinement of folding techniques and workmanship precision, bookend shape, preload, and quantity, along with integration of secondary sequencers were pursued over a series of 6 developmental deployments. A fundamental difficulty was that the loads carried thru the sail (as the outboard corners are raised to the mast tips by the halyards) were excessive. Initially, the sail folds did not uniformly release from the book clips due to variations in friction and the amount of sail engagement in the clips. These variations would result in a shearing action that caused the secondary sequence elements to release before the stack sequence was complete. Making "stronger" book clips was not an option. Our goal was to insure the limiting load on the mast was the deployed sail tension, not the deployment of the sail. This allows optimization of mast mass for large sail systems. On the other hand, a weak clip release load could result in a premature or multiple fold release. Such action would leave portions of the sail uncontrolled, presenting a potential for mast contact.

Irrespective of arguments whether contact with the mast could or would result in snagging of the sail, it became clear that a robustly-managed deployment would not be attainable with a book clip approach. A new method for storage and deployment control was introduced which presents several advantages. First it avoids the difficulties of secondary folding. Secondly, the sequencing approach assures maximum separation between the sail and the mast. Thirdly, the mast load during deployment is kept below the deployed loading. Lastly, the methods for packaging are easily scalable to nearly unlimited dimensions.

In this new method, depicted in Figure 9, the primary (15-in.) folding is done as described above. Next the sail is folded in half, and rolled onto a drum. Cross-ties to hold the halves together during the first stage of deployment provide for the greatest possible separation of the sail and masts during deployment.

As the sail is pulled out during the first stage of

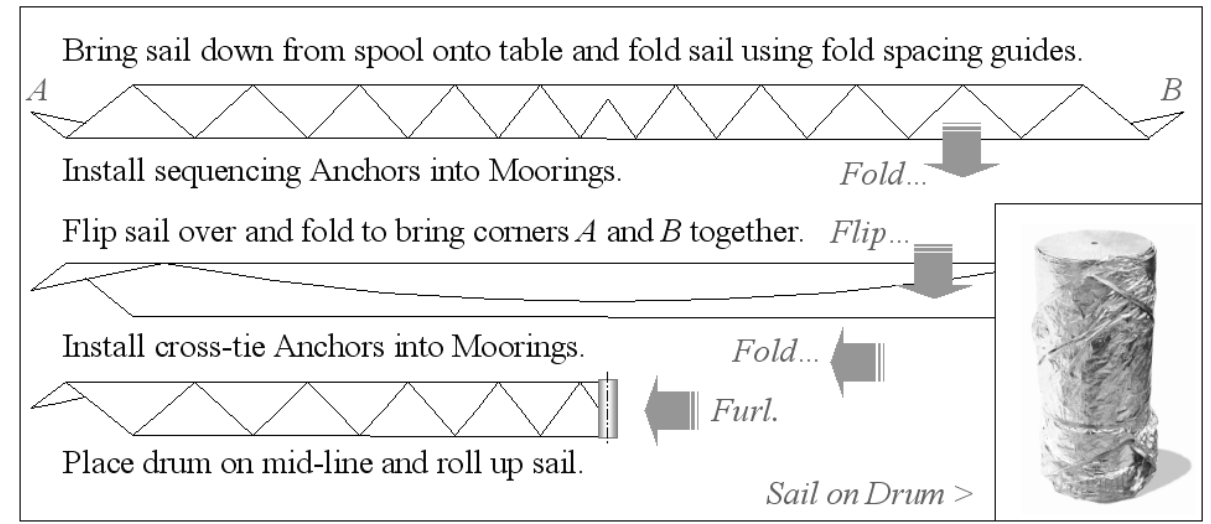

Figure 9. Sail Stowage Sequence: Fold and Furl 
deployment, the load is kept light, but fixed, by a constant drag torque mechanism in the drum. The general deployment sequence is depicted in the series of photos captured on January $30^{\text {th }}$ (for 3 photos to the right) during the first drum deployment trials.

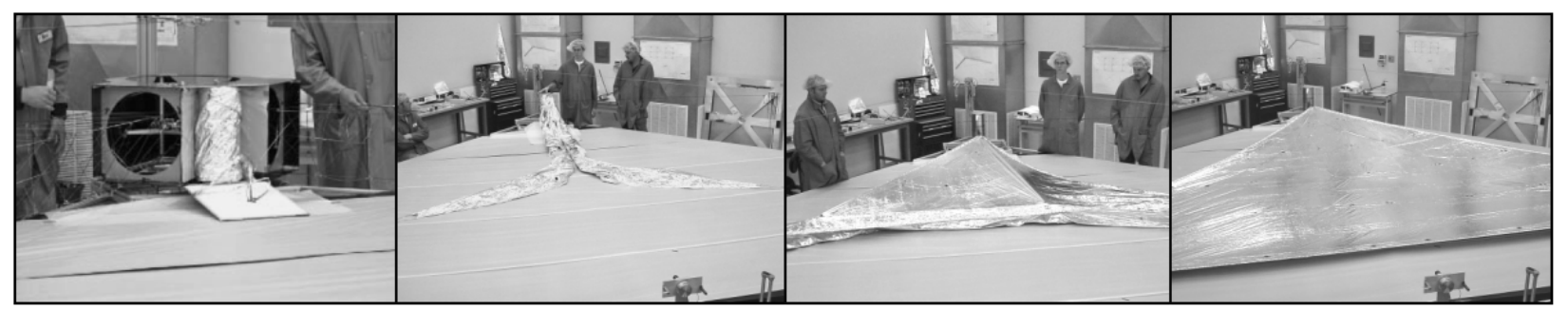

Figure 10. Managed Sail Deployment Sequence

The elements that enforce the sequential deployment are lightweight and resettable. The placement and design of the sequencers is depicted in Figure 11. One or more "anchors," affixed to the sail through a fabric line bonded to a "cleat," is engaged into the adjacent "mooring" during the fold process. The cleats and moorings are bonded into the sail with a doubler ring of CP1 sail material and pressure sensitive adhesive (PSA). The moorings and cleats are made of translucent white engineering thermoplastic. Prior to the placement of these elements, a sail material retain is collected by punching out a $1 / 2$-in. sample from the sail membrane.
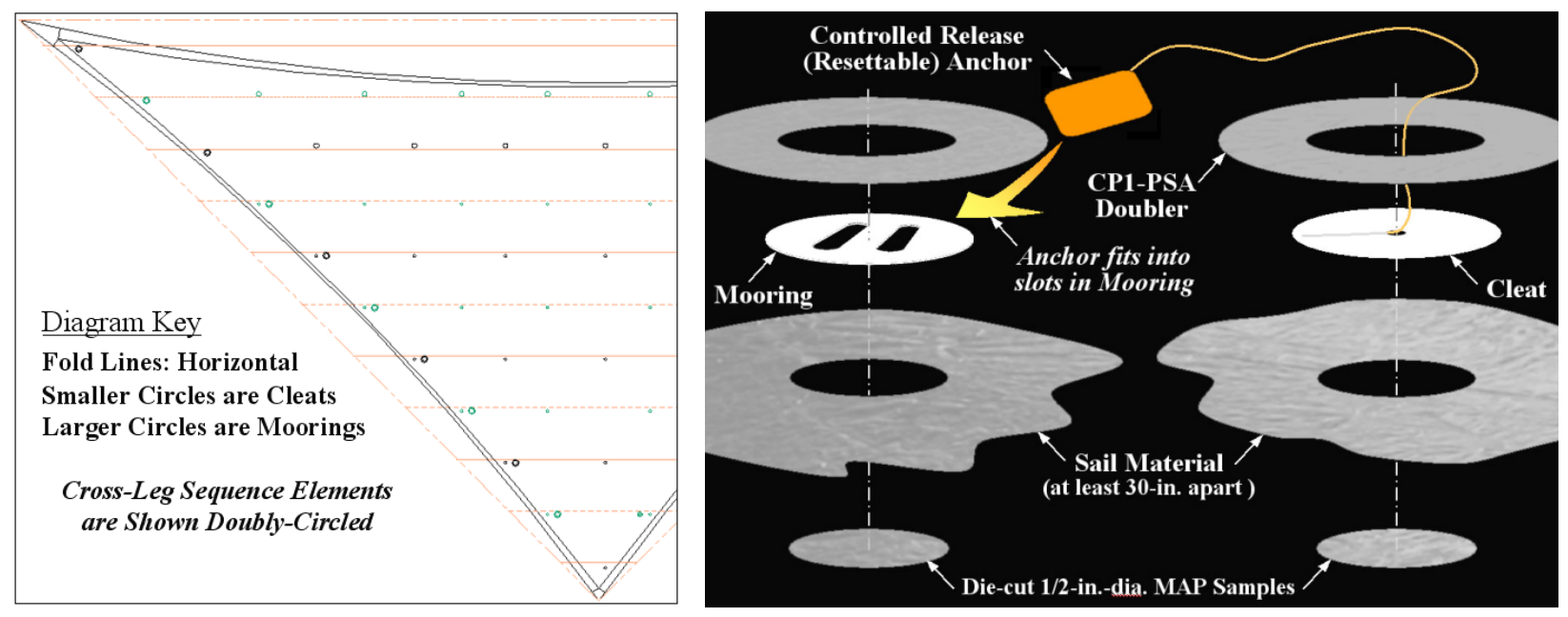

Figure 11. Fold Pattern and Generic Sequencers Design (with Target \& MAP Retain Utility)

A unique and redundant sequencer holds the sail halves together at the outboard grommets as the sail is pulled off the drum. The two halves separate into a Y pattern as the cross-tie sequencers release. Once the halves are separate, the sequencers in the field of the sail allow unfolding of the 15-in. folds one at a time starting at the inboard fold line and progressing out.

As can be seen to the left in Figure 11, the sequencers form a fairly regular pattern across the sail. The purpose of using translucent white plastic for the sequencer elements is that the retain punch-out allows sunlight to be diffused through these elements, which creates an excellent target for optical evaluation of sail shape and dynamics from the anti-sun side of the sail. The $\mathrm{S}^{4}$ design incorporates the option for various-length offset booms to deploy instruments fore and aft of the sail. For the flight validation mission a boom deployed aft (to the dark side) of the sail will allow imaging of these targets. This vantage point avoids the issues of large-scale glare and small-scale glint (from creases and wrinkles) that viewing from the sun side imposes. From the aft side, the sail will be relatively dark as the sail coating is opaque. The sunlight diffused thru the sequencer forms a distinct and bright image, discernable by a camera even if viewed at a low angle to the sail. An example of a sequencer/target in a sail sample, illuminated from behind, is shown in Figure 12.

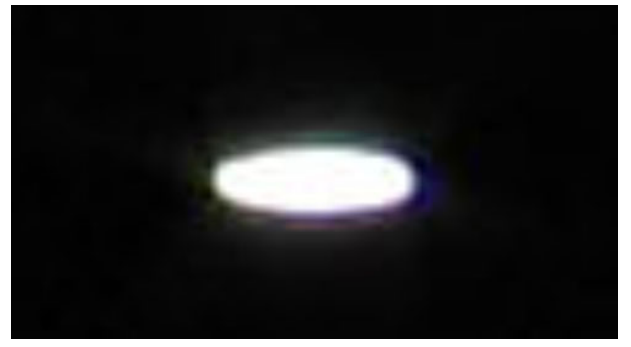

Figure 12. Sample Target Image at $20^{\circ}$ 
The function of the retain collection is to allow Mass and Absorptivity Properties (MAP) sampling from which to construct a mathematical model of the as-built sail mass properties (e.g. center-of-gravity (CG)) and center-ofpressure (CP). The CP-CG offset is the critical design driver in the sailcraft ACS design. Without accurate MAP data, significant risk is present that the authority of control over the CP-CG imbalance disturbance will be overwhelmed in flight. The relatively large, uniform material sampling provided by the retains will effectively minimize any CP-CG uncertainty due to sail property variability. This data is also useful for detailed inertia modeling for sail and system dynamics.

The development of the new baseline packaging and sequenced-deployment process required a significant effort that was not fully refined during ambient testing due to schedule limitations, but a basic robust functionality was achieved which was the most critical goal of development and test of the system.

After completion of the mast and sail component testing regimen (refer to Figure 4), the integration of the masts, sail, and capstan mechanism to the central assembly and offloading GSE, followed by electrical and physical inspections and several system functional (deployment) trials, the 10-m Quadrant Assembly was ready for shipment to Virginia for testing in a LaRC vacuum chamber.

\section{Vacuum Testing Program}

\section{A. Overview}

System testing in vacuum is necessary because both the deploying and deployed dynamics of a sail could otherwise be greatly affected by the surrounding air mass. As a illustration, the air within $2 \mathrm{~mm}$ of either side of the sail surface is alone equal in mass to a 3-micron sail film. In order to validate deployment characteristics as well as sail shape and system dynamics, as series of tests were planned utilizing the 16-m vacuum chamber at NASA LaRC. The priorities for testing were first to validate deployment, second to measure deployed shape of the (horizontal) sail billowed under gravity loading, and third, to validate models for system dynamics.

The ABLE/LaRC test team planned an extensive series of tests to capture the data needed to support these priorities, as well as to meet goals for developing test methods applicable to larger scale testing and to in-flight investigation. The testing sequence is shown in Figure 13. The LaRC test team vigorously pursued development and validation of dynamic test methods ${ }^{7}$ and other readiness preparations using EDU hardware (sails and mast) prior to formal testing over a period of months leading up to Quadrant Testing.

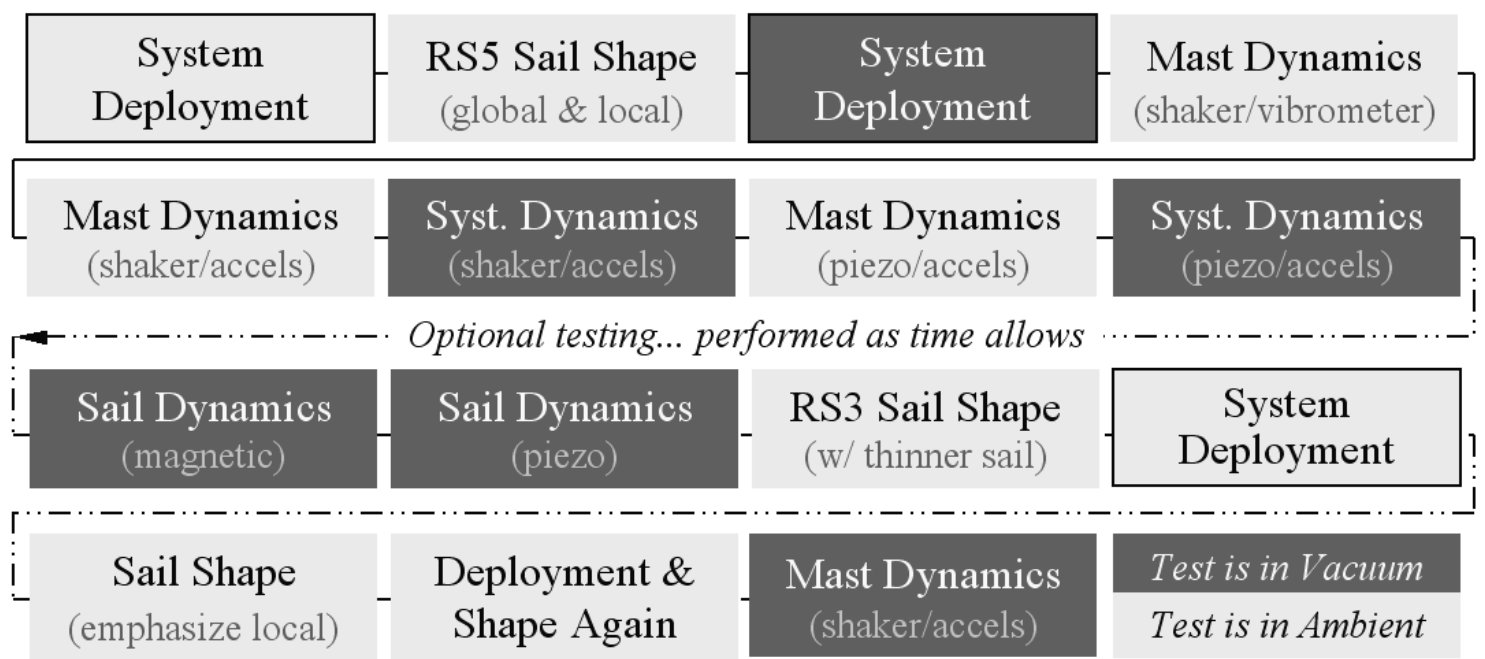

Figure 13. 10-m S $\mathrm{S}^{4}$ Quadrant Assembly Test Plan Flow

\section{B. Progress to Date}

Testing at LaRC with the 10-m Quadrant began on March 30, 2004 with a successful ambient deployment of the system to verify the GSE setup after shipment and installation in the vacuum chamber. One of the sequencing elements failed to release and the sail was torn locally. It was concluded that the (handmade prototype) anchor was able to rotate and jam within the mooring due to imprecise fabrication. All anchors on RS5 were subsequently replaced with precision-cut elements of a thinner material to assure reliable functionality and to increase load margins. The RS3 sail is fabricated with die-cut sequencer elements to preclude the potential for snagging. 
With the system deployed in a horizontal condition, measurement of sail shape was first attempted. Knowledge of shape is critical for two reasons. Firstly, global shape affects the modal response of the sail. Secondly, fine scale topology is critical the thrust performance. The LaRC facility is equipped with a laser radar scanner system that can measure the entire sail surface rapidly with a high accuracy. But testing progress was immediately stalled by two difficulties: The scanner had difficultly capturing a return off the highly reflective sail material, and the sail was not staying still. Both of these difficulties were not anticipated because previous testing with similar sails had been successful. Methods to scan the more reflective (and thinner) RS5 sail were quickly developed. The motion of the sail within the vacuum chamber proved difficult to diagnose, as the motions were not repeatable in amplitude, frequency or time of day. Vibrations from nearby equipment (such as wind tunnels, AC units, sump pumps, and chillers) were suspected. The deployment off-loading surface sheets were removed to investigate if mechanical vibrations were driving air motion between this surface and the sail.

A second ambient deployment was conducted (April $6^{\text {th }}$ ) to validate the sequencing anchor rework. As the delay in testing caused by the anomalous sail motion behavior became significant, it was determined that proceeding with the vacuum deployment test was the best course of action. After the vacuum chamber had stabilized near 1 torr, the sail did remain still. Yet, this data did not serve as confirmation that sail motion was caused by air effects, as even in an ambient environment there were large parts of several preceding days where the sail was still as well.

The deployment of the system in vacuum was entirely successful. Data on mast and sail deploy speed, motor current (convertible to lanyard and halyard loads), and tack line tension were recorded, as well as imagery from two fixed position digital cameras and three pan-zoom video cameras. An image from one of the digital cameras is shown in Figure 14. The gossamer masts are hardly visible here (refer back to Figure 1), but the distribution of 111 photogrammetry targets is evident. With adjusted lighting, such imagery will be used to measure global shape.

The digital cameras are in vacuum-compatible housings, whereas the laser radar is not. Therefore, the option of measuring detailed sail shape while in vacuum was not available in the short term. It was determined that the source of the sail motion was chaotic low-velocity airflow precipitated by thermal stratification in the vacuum chamber.

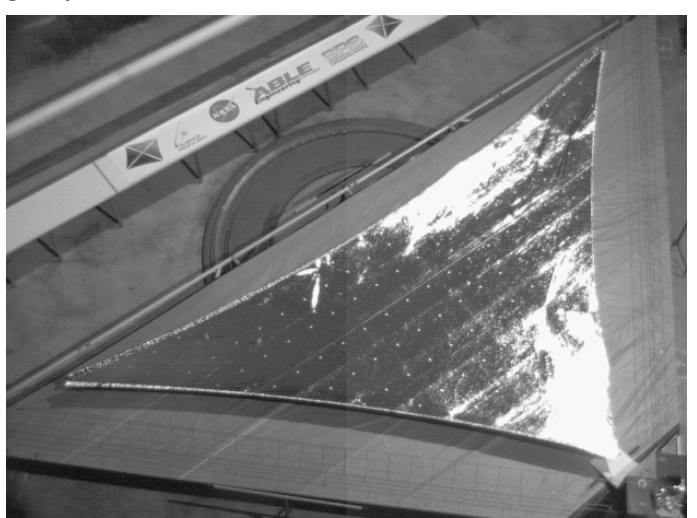

Figure 14. 10-m $\mathrm{S}^{4}$ Quadrant in Vacuum Post Deployment (April 7, 2004)

Depending on the time of day and weather conditions, the air within the chamber would circulate almost imperceptibly, but given the large horizontal expanse of lightweight material, this was enough to make the sail "breathe" once or twice a minute, at times with amplitudes of 1-3 centimeters.

Tests with a separate sail in a nearby high-bay confirmed that shape testing there (with the AC off) was feasible. Testing of the sail, suspended by new GSE, using the laser radar was then initiated in parallel with mast dynamics testing in the vacuum chamber. This work-around plan allowed the testing team to return to the original schedule for the beginning of mast dynamics on Friday the $12^{\text {th }}$, with the potential for reintegration of the sail with the masts in the vacuum chamber for system testing by the end of the following week.

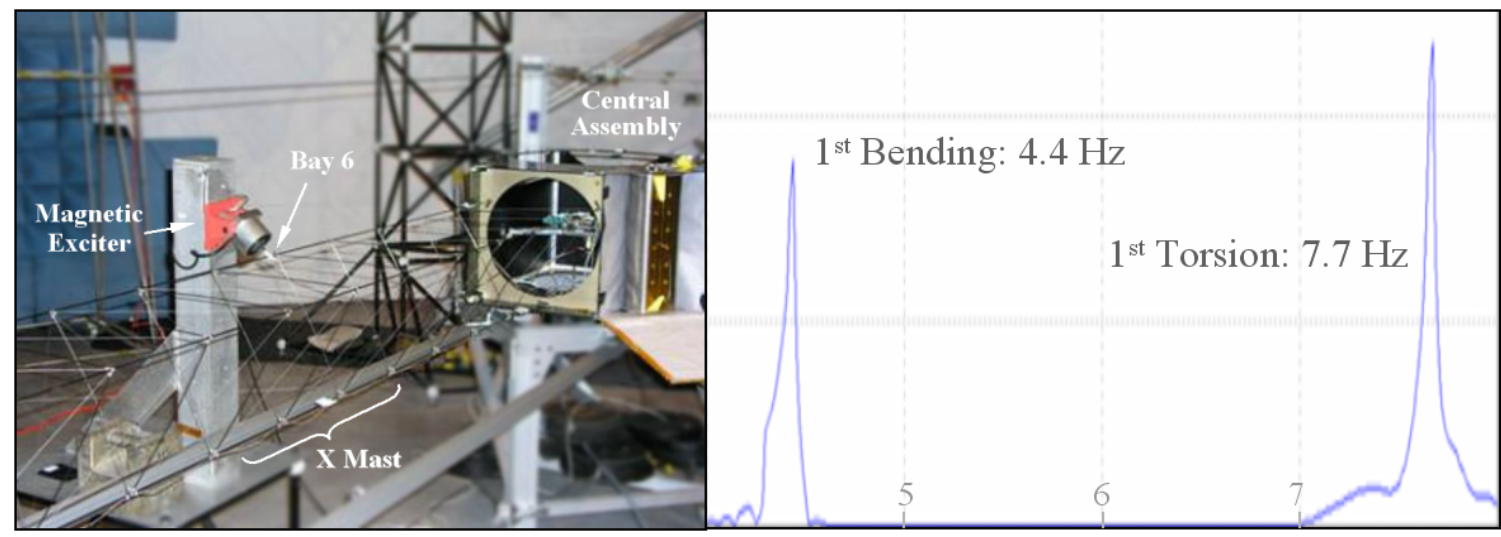

Figure 15. Mast Dynamics Test Set-up Using Magnetic Excitation and First-Look Frequency Response 
Mast dynamic testing began with the installation of a magnetic excitation device at $45^{\circ}$ to the inboard longeron of the $\mathrm{X}$ mast at the $6^{\text {th }}$ bay. Using laser vibrometer targets at the mast tip, the in-plane and out-of-plane first bending mode and the first torsional vibration mode were captured. The test setup and modal results are shown in Figure 15.

Greater mass distributed along the mast (harnessing and vibrometry targets) and at the tip, in combination with the central structure and GSE flexibility, resulted in lower mast modes (4.4 vs. $5.7 \mathrm{~Hz})$ than were recorded during earlier component level testing at ABLE, or predicted $(4.7 \mathrm{~Hz})$ for the integrated masts. This first-look data for mast modal response agrees fairly well with pre-test predictions as shown at the left in Figure 16. The first mode of the sail is much lower, so to accelerate system model convergence the GSE and Central Structure flexibilities were incorporated into the mast model to produce predictions such as shown to the right in Figure 16.

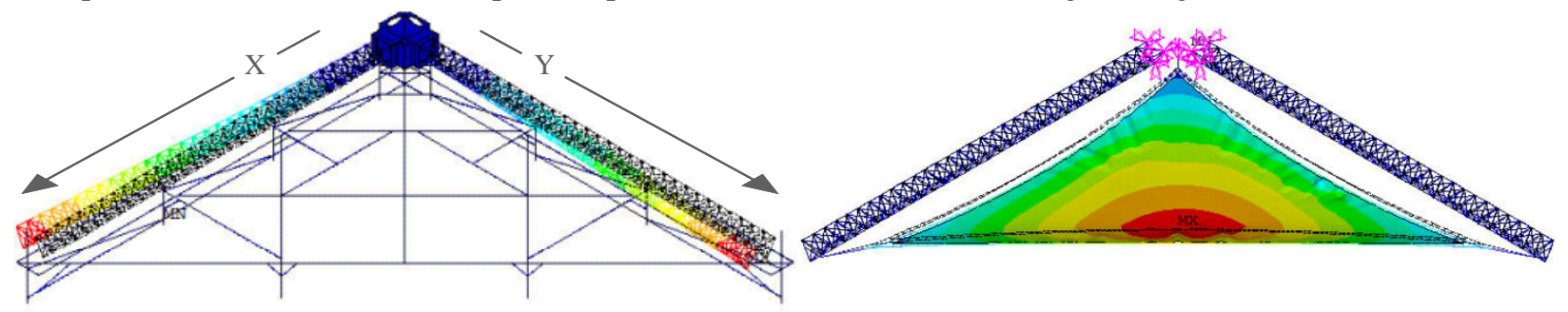

Figure 16. Mast-Central Assembly-GSE and System FEA Model Results, ${ }^{\text {st }}$ Modes: 4.7 and $1.5 \mathrm{~Hz}$

Testing of the system in ambient and in vacuum, using a variety of input methods and output methods, is currently in progress. Traditional vibration input methods (mechanical and magnetic shakers) are baselined to validate a developmental method (Piezo actuators applied to the mast longerons) that has potential for use in flight. The baseline output method is vibrometry and the flight-applicable developmental method utilizes MEMS accelerometers distributed along the masts.

At the conclusion of system dynamics testing, a second round of global and local sail shape measurements will be made on the RS3 sail, which incorporates the upgraded sequencer design described earlier, that has dual functionality as photogrammetry targets.

\section{Ongoing Activities}

As further test data is gathered in the later half of April, sail shape and system dynamic model correlation activities will commence. The second set of shape data, on the thinner (3-micron) RS3 sail will provide additional opportunity for detailed correlation activities with the shape prediction models, which after the completion of vacuum deployment demonstration, is now our highest priority. Given correlated (and scalable) models to predict membrane billow and wrinkling in $1 \mathrm{~g}$, predictions of the dynamics (third priority) can be made with confidence. The ability to confidently predict the general shape and approximate frequencies of the first few system modes is essential for control system design for flight.

In parallel, work has recently begun on the design of the $20-\mathrm{m} \mathrm{S}^{4}$ system, which will be tested in the 100-ft diameter Plum Brook vacuum chamber facility in April of 2005. The 20-m design will be a full system (4 quadrants, 4 masts, instrument offset boom), including flightrepresentative structure for the central assembly, additional mechanism for deployment and attitude systems control, tiedown/release hardware, solar panels, and payload adaptor fairing interfaces, to comprise a bus chassis, photon propulsion system, and sailcraft ACS. The demonstration of this system will elevate the TRL of solar sailing technology in all areas critical to support a lowrisk flight validation mission in earth orbit.

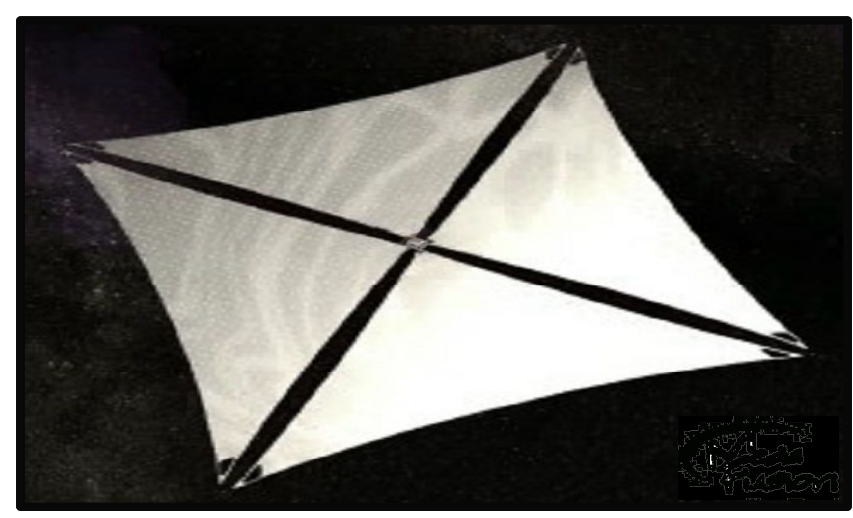

Figure 17. Graphic of $S^{4}$ System in Flight 


\section{Summary}

The soundness of design of a deployable integrated sail and structure system has been validated with an extensive series of tests. Analytical correlation activities completed to date have shown excellent agreement with test results. These activities are supporting continuing system design and analytical development that will ensure scalable design and accurate predictions for larger ground systems, a flight demonstration system (40-m scale), and mission systems of up to 200 meters on a side. Further validation activities are currently ongoing in parallel with the design and test planning for demonstration of a full (4-quadrant) 20-m sail system.

\section{Acknowledgments}

The efforts reported on herein are sponsored under NASA Contract NAS8-03043. This paper was written to maximize dissemination of information derived from this activity to the benefit of the national space program. The authors wish to thank Sandy Montgomery, Greg Garbe, and the ISP projects staff for their leadership and support and to recognize the vital design engineering contributions in this demonstration phase provided by Peter Barker, Mark Douglas, Greg Laue, Mike McEachen, and Arial Pavlick.

\section{References}

${ }^{1}$ Garbe, G., Montgomery, E., Heaton, A., Van Sant, J., and Campbell, B., "NASA's Integrated Development of Solar Sail Propulsion," AAS 04-103, 14 $4^{\text {th }}$ AAS/AIAA Space Flight Mechanics Meeting, 2004.

${ }^{2}$ Murphy, D., Murphey, T., and Gierow, P., "Scalable Solar-Sail Subsystem Design Concept", AIAA Journal of Spacecraft and Rockets, Volume 40, No. 4, pp. 539-547, July-August 2003.

3 Murphy, D., Trautt, T., McEachen, M., Messner, D., Laue, G., and Gierow, P., "Progress and Plans for System Demonstration of a Scalable Square Solar Sail," AAS 04-105, 14 ${ }^{\text {th }}$ AAS/AIAA Space Flight Mechanics Meeting, 2004.

4 "The Sun-Earth Connection Roadmap 2003-2028, Understanding the Sun, Heliosphere, and Planetary Environments as a Single Connected System," NP-2002-8-500-GSFC. Available at http://sec.gsfc.nasa.gov

${ }^{5}$ Murphy, D., and Wie, B., "Robust Thrust Control Authority for a Scalable Sailcraft, AAS 04-285, 14 $4^{\text {th }}$ AAS/AIAA Space Flight Mechanics Meeting, 2004.

${ }^{6}$ Crawford, R., and Benton, M., "Strength of Initially Wavy Lattice Columns", AIAA Journal, Vol. 18, No. 5, pp. 581-584, May 1980, Presented as Paper 79-0753 at the AIAA Structures Dynamics and Materials Conference, 1979.

${ }^{7}$ Gaspar, J., Mann, T., Behun, V., Wilkie, K., and Pappa, R., "Development of Modal Test Techniques for Validation of a Solar Sail Design”, 45 ${ }^{\text {th }}$ AIAA Structures, Structural Dynamics, \& Materials Conference, $5^{\text {th }}$ Gossamer Spacecraft Forum, 2004. 